\title{
Advances in Methods for Control over Networks
}

\author{
Ya-Jun Pan, ${ }^{1}$ Peter X. Liu, ${ }^{2}$ Yang Shi, ${ }^{3}$ and Jie Sheng ${ }^{4}$ \\ ${ }^{1}$ Department of Mechanical Engineering, Dalhousie University, P.O. Box 15000, Halifax, NS, Canada B3H 4R2 \\ ${ }^{2}$ Department of Systems and Computer Engineering, Carleton University, 1125 Colonel By Drive, Ottawa, ON, Canada K1S 5B6 \\ ${ }^{3}$ Department of Mechanical Engineering, University of Victoria, P.O. Box 3055, STN CSC, Victoria, BC, Canada V8N 3P6 \\ ${ }^{4}$ Institute of Technology, University of Washington Tacoma, 1900 Commerce Street, Campus Box 358426, Tacoma, \\ WA 98402-3100, USA
}

Correspondence should be addressed to Ya-Jun Pan, yajun.pan@dal.ca

Received 3 June 2012; Accepted 3 June 2012

Copyright (C) 2012 Ya-Jun Pan et al. This is an open access article distributed under the Creative Commons Attribution License, which permits unrestricted use, distribution, and reproduction in any medium, provided the original work is properly cited.

Control over networks is one of the two main research areas in networked control systems, where systems to be controlled, actuators, sensors, and controllers share and exchange information over wire-linked or wireless networks rather than hardwired. The system elements are typically spatially isolated from one another and operating in an asynchronous manner. With the introduction of communications networks in control systems, researchers from a variety of disciplines in both industrial and university research centers have become interested in this exciting and challenging research area due to its wide applications. Several common constraints in the networked control systems are (i) transmitting delays in transferring information to and from the controller; (ii) limited communication bandwidth for the feedback information being sent to the controller and for the control commands being transmitted to the actuators; (iii) data dropout due to network congestions.

In the past decade, the research in this area was active and gained significant progress with many successful applications; however, tremendous theoretical and technical challenges still exist. Many fundamental issues such as stable operation of interconnected real-time systems, signal coding, decoding and control information flow, and effects of the network constraints on the performance of control systems, remain to be dealt with.

The main focus of this special issue is on the most recent theoretical developments, results, and applications in the area of methods for control over networks. A total of 14 papers were submitted for this special issue, and 7 out of the submitted papers have been accepted for this special issue and present interesting results.
The special issue is initiated by a paper written by $\mathrm{X}$. Meng and T. Chen, which focuses on the asymptotic stabilization of linear systems over networks based on event-driven communication with time-varying delays. A new communication logic is proposed to reduce the feedback effort providing more advantages over traditional ones with continuous feedback.

L. Sheng et al. propose a consensus-based formation control for a class of networked multiple mobile robots with a virtual leader approach. A multiple Lyapunov Krasovskii functional candidate is proposed for investigating the sufficient conditions to linear control gain design for each robot. Simulation results and experimental studies on Pioneer 3 series mobile robots are shown to verify the effectiveness of the proposed approach.

When there are sensor nonlinearities and packet loss in the system, a robust $H^{\infty}$ filtering approach of a class of discrete-time stochastic system with randomly occurred nonlinearity is investigated by T. Sun et al. Based on the Lyapunov-Krasovskii functional method, the asymptotical mean-square stability condition of the filtering system with a prescribed $H^{\infty}$ level is derived.

The packet loss issue is further dealt with in the paper by C. Hsieh and P. Hsu. Dropout data can be properly recovered by applying different message estimators to improve motion contouring accuracy. The short-window dropout quantity is proposed to estimate the network quality based on the dropout rate, and its distribution of the missing data and switching least-square estimator are proposed to compensate for missing motion commands. 
A CAN-based networked control system is applied to a multifunctional robotic platform in the work by E. Godoy et al. for precision agriculture. The results demonstrate that the NCS was simple and efficient, providing suitable steering performance for the platform guidance, and overcame some verified control challenges in the robot guidance system design such as the hydraulic system delay, nonlinearities in the steering actuators, and inertia in the steering system due to the friction of different terrains.

Under the random packet loss in both channels, a robust fault-tolerant controller design problem is investigated by $\mathrm{X}$. Qi et al. The system includes state delay, model uncertainty, disturbance, probabilistic sensor failure and actuator failure. A sufficient condition for the asymptotic mean-square stability of NCS is derived with a designed fault-tolerant controller.

Finally, J. Wang et al. study the stabilization problem of a wireless networked control system with time delays and packet loss existing simultaneously. The system is modeled as an asynchronous dynamic system (ADS) with unstable subsystems. A sufficient condition for the system to be stable is presented and demonstrated by a numerical example.

We expect that the papers included in the special issue contribute significantly to advance the research on the methods for control over networks. Finally, we would like to thank all the authors who have submitted their papers to the special issue and all the reviewers who helped handling the submitted papers to the special issue.

Ya-Jun Pan

Peter X. Liu

Yang Shi

Jie Sheng 

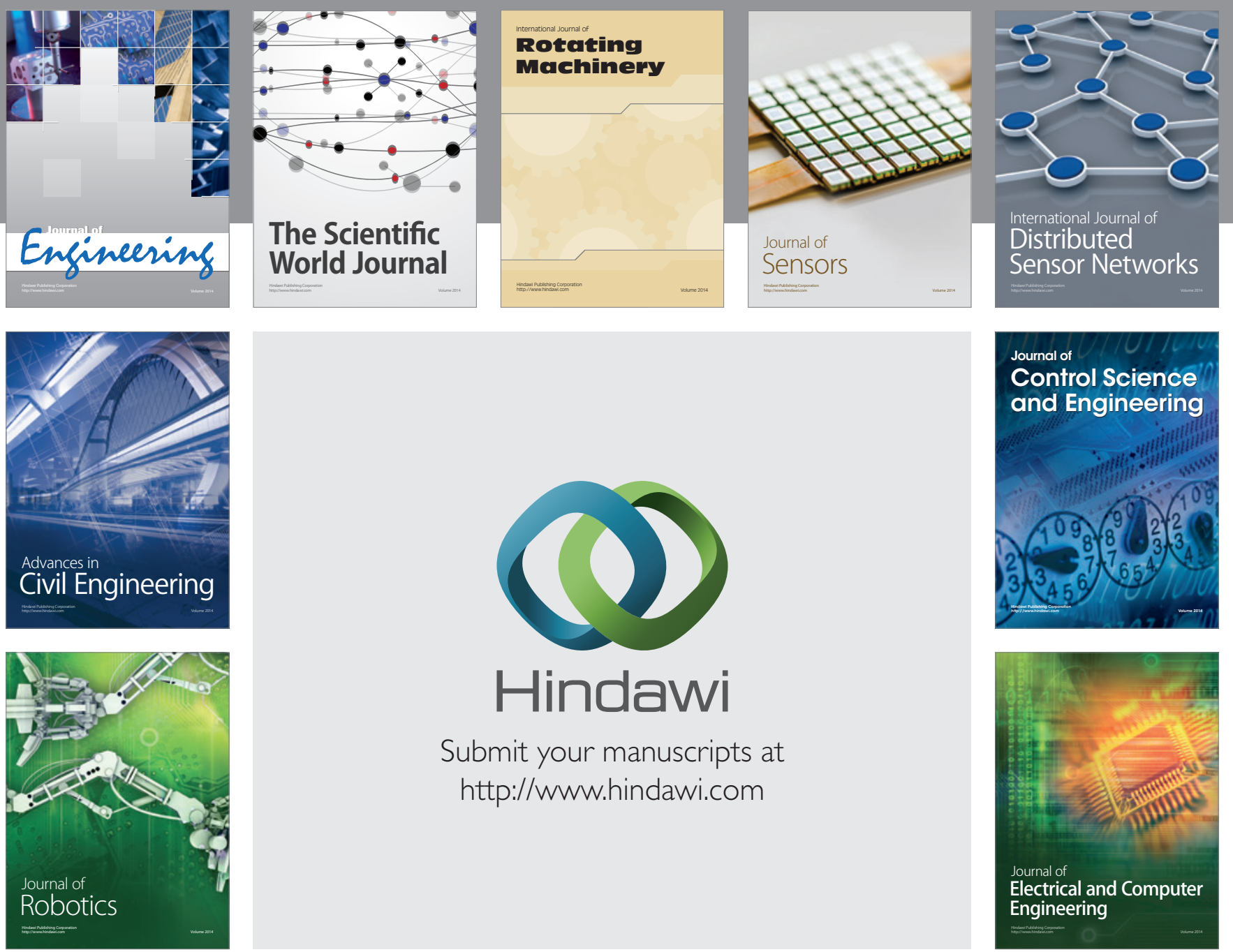

Submit your manuscripts at

http://www.hindawi.com
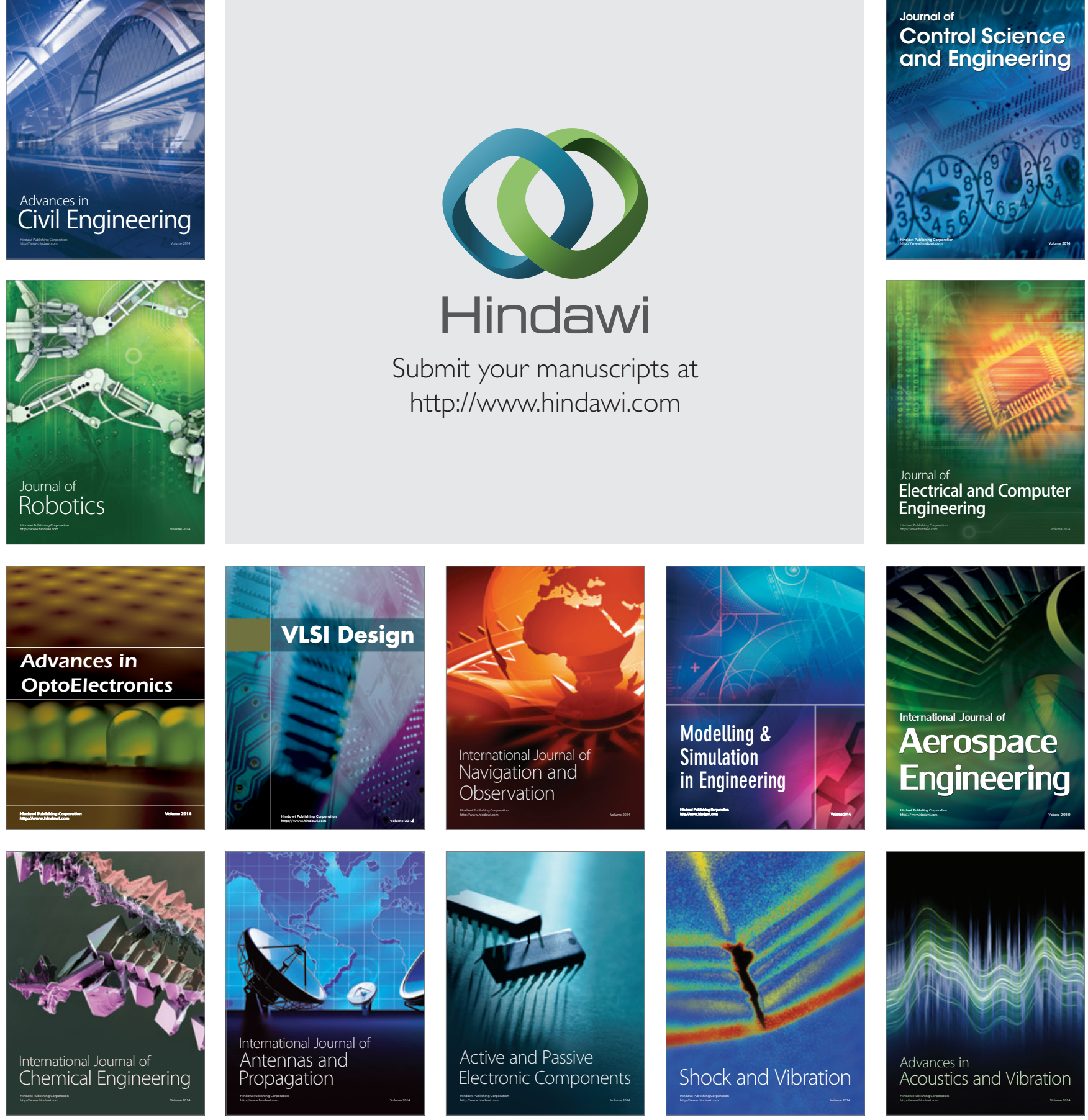